\title{
IMPORTÂNCIA DOS ANNELIDA - POLYCHAETA PARA A BIOMASSA BENTICA DA PLATAFORMA CONTINENTAL DAS ILHAS KERGUELEN
}

Maria Alice de Carvalho Reis ${ }^{1}$

\begin{abstract}
Using material collected by the Oceanographic Expedition MDO4 in the Continental Shelf of Kerguelen Islands, Subantarctic Region, we focused the dominance and the density of the different zoological groups found to be present in relation to the Annelida Polychaeta.
\end{abstract}

\section{INTRODUÇÃO}

$\mathrm{O}$ estudo do bentos da plataforma continental das Ilhas Kerguelen e do ecossistema Subantártico ou Austral coloca em evidência a importância da biomassa do zoobentos da região, cujo índice é considerado um dos maiores do mundo.

Estudos recentes comprovam que as comunidades animais e vegetais são bastante densas em torno dessas ilhas. Esta densidade parece estar ligada a fatores físicos, aos movimentos das massas d'água, a nível de Convergência e Divergência Antárticas e aos sais nutrientes.

De acordo com Desbruyères \& Guille, 1973, a biomassa bêntica da costa Kerguelenense apresenta-se muito elevada, superior mesmo às assinaladas em idênticas condições no Oceano Glacial Antártico e bem mais importante que as conhecidas em mares temperados e tropicais.

Após os trabalhos de Arnaud, 1974, a maioria das pesquisas consagradas à composição e à distribuição das populações de substrato móvel foi fundamentada na macrofauna, do ponto de vista quantitativo e qualitativo, mas os resultados sobre biomassa de fundo móvel, em sua maior parte, estavam concentrados apenas no Golfo de Morbihan.

Desbruyères \& Guille, 1973, Desbruyères, 1977, Guille, 1977, Hureau, 1973, Bellan, 1975 e De Bovée, 1976, assinalam que as Ilhas Kerguelen oferecem possibilidades singulares de contribuição à economia marinha Subantártica, devido também à presença de uma plataforma continental extensa.

Não é pois surpreendente que o estudo quantitativo e qualitativo do macro e meio bentos tenha despertado o interesse de pesquisadores e constitua um amplo programa de estudo multidisciplinar.

1 Centro de Ciências Biológicas, Departamento de Biologia Animal, Universidade Santa Ursula - RJ. 
Com efeito, pesquisas do macrobentos, sob o ângulo sinecológico e sistemático, inventário numérico e ponderal da fauna, reconhecimento e delimitação dinâmica das comunidades, auto-ecologia, adaptação ecofisiológica ao meio Subantártico, a nível de reprodução de crescimento relacionado ao fenômeno de gigantismo e nanismo alcançaram um grande incremento.

Não retomaremos aqui a totalidade dos trabalhos concernentes ao macrobentos da plataforma continental da costa de Kerguelen, mas sínteses notáveis realizadas por autores como Arnaud, 1974, Guille \& Soyer, 1976, Desbruyères, 1976b, Desbruyères \& Guille, 1976.

São muito fragmentários, no entanto, os conhecimentos sobre os Anelídeos Polychaeta da plataforma continental das Ilhas Kerguelen, largamente dominantes qualitativa e quantitativamente na comunidade bêntica. Desbruyères \& Guille, 1973, Desbruyères, 1976a, b, Chardy et alii, 1976 procuraram definir as populaçб̃es Anelidianas, mas todas as pesquisas se limitaram ao Golfo de Morbihan.

Reconhecendo, pois, a importância dos Polychaeta no ecossistema da região Subantártica, escolhemos direcionar para aí nossa pesquisa.

\section{ILHAS KERGUELEN}

O Arquipélago de Kerguelen, formado por 400 ilhas, está situado ao Sul do Oceano Indico, nas Terras Austrais Francesas, a 49015' Latitude Sul e 69033' Longitude Leste, entre a çonvergência Antártica e a Subtropical (Fig. 1).

\section{MATERIAL E MÉTODOS}

O material estudado é proveniente da Campanha Oceanográfica MDO4, realizada de 14 de fevereiro a 24 de março de 1975 na plataforma Continental das Ilhas Kerguelen; pertence à Dra. Janete Maron Ramos e encontra-se na Universidade Santa Ürsula-RJ.

Foram projetadas 121 estações, distribuídas por um total de 10 radiais (Fig.2). Tais radiais estão localizados ao redor das Ilhas Kerguelen.

De acordo com a batimetria, as estações foram assim distribuídas:

$$
\begin{aligned}
& \text { Menos de } 20 \mathrm{~m} \text { de profundidade - } 5 \text { estações } \\
& \text { de } 20 \mathrm{a} \quad 50 \mathrm{~m} \text { de profundidade }-17 \text { estações } \\
& \text { de } 50 \text { a } 100 \mathrm{~m} \text { de profundidade }-20 \text { estações } \\
& \text { de } 100 \text { a } 200 \mathrm{~m} \text { de profundidade }-43 \text { estações } \\
& \text { de } 200 \text { a } 300 \mathrm{~m} \text { de profundidade }-19 \text { estações } \\
& \text { de } 300 \text { a } 400 \mathrm{~m} \text { de profundidade }-10 \text { estações } \\
& \text { Mais de } 500 \mathrm{~m} \text { de profundidade }-8 \text { estações }
\end{aligned}
$$

A duração média de cada estação foi de aproximadamente 2,45 h. Das 144 estações efetuadas com a Draga Charcot-Picard (DC), 70 foram por nós estudadas no plano quantitativo devido à presença de Annelida-Polychaeta.

As amostras de água foram coletadas a profundidade-padrão com garrafas tipo Niskin, em PVC, com capacidade de 1,8 litros, equipados com termômetros.

As temperaturas foram medidas com termômetros protegidos e não protegidos Richter \& Wiese, permitindo uma precisão de aproximadamente $0,001{ }^{\circ} \mathrm{C}$. 


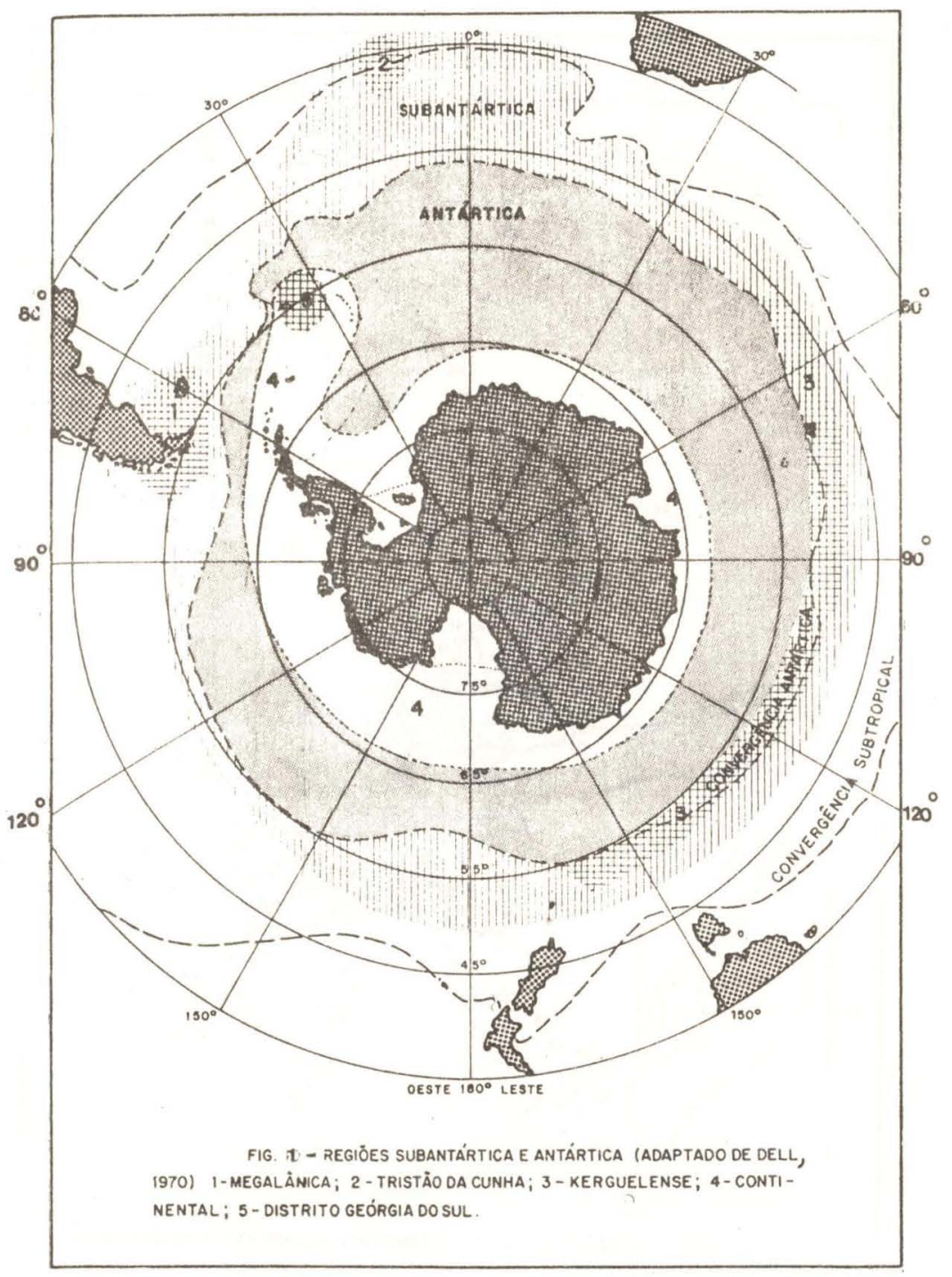




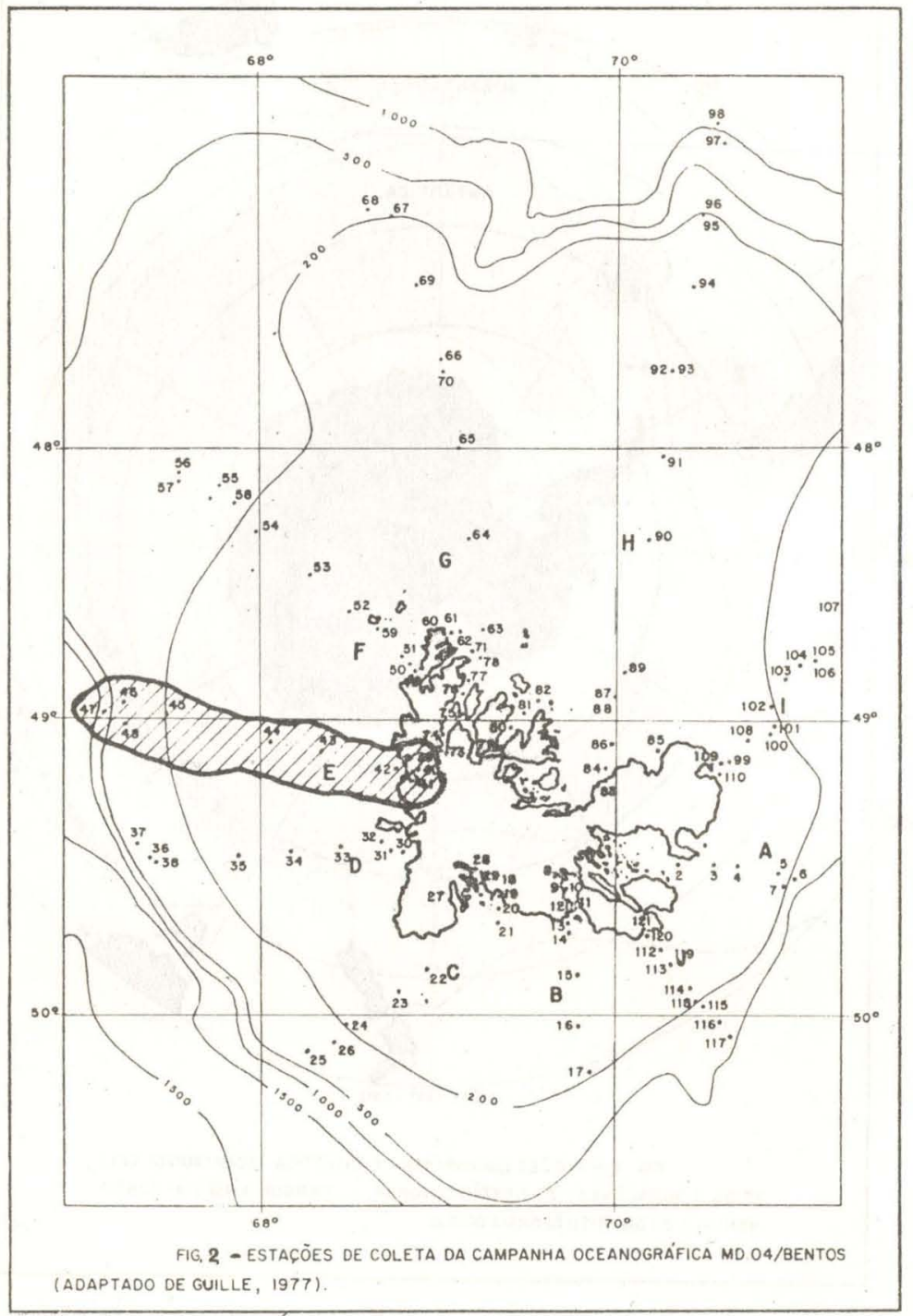


FIC. 3 - DADOS QUANTITATIVOS RELATIVOS AO: MACROBENTOS DE KERGUELEN

\begin{tabular}{|l|c|c|c|c|c|c|}
\hline ESTAÇÕES & \multicolumn{2}{|c|}{ A 02 } & \multicolumn{2}{c|}{ A 0? } & \multicolumn{2}{c|}{ A 04 } \\
\hline PROFUNDIDADE (m) & \multicolumn{2}{|c|}{$32-44$} & \multicolumn{2}{c|}{75} & \multicolumn{2}{c|}{96} \\
\hline PERCENT. DENS. & $\%$ & ind. $/ \mathrm{m}^{2}$ & $\%$ & ind. $/ \mathrm{m}^{2}$ & 7 & ind. $/ \mathrm{m}^{2}$ \\
\hline Polychaeta & 53.63 & 362 & & & & \\
\hline Crustacea & 19.09 & 126 & & & & \\
\hline Mollusca & 8.48 & 56 & & & & \\
\hline Nematoda & 8.78 & 58 & & & & \\
\hline Echinodermata & 3.33 & 22 & & & & \\
\hline Diversos & 6.66 & 44 & & & & \\
\hline & & & & & & \\
\hline
\end{tabular}

\begin{tabular}{|l|c|c|c|c|c|c|}
\hline ESTAÇÕES & \multicolumn{2}{|c|}{ A 05 } & \multicolumn{2}{c|}{ A 06 } & \multicolumn{2}{c|}{ B 08 } \\
\hline PROFUNDIDADE (m) & \multicolumn{2}{|c|}{140} & \multicolumn{2}{c|}{$357-542$} & \multicolumn{2}{c|}{21} \\
\hline PERCENT. DENS. & $\%$ & ind. $/ \mathrm{m}^{2}$ & $\%$ & ind. $/ \mathrm{m}^{2}$ & $\%$ & ind. $\mathrm{m}^{2}$ \\
\hline Dolych aeta & 40.44 & 72 & & & 25.41 & 552 \\
\hline Crustacea & 5.61 & 10 & & & 20.07 & 436 \\
\hline Mollusca & 35.95 & 64 & & & 40.97 & 890 \\
\hline Nematoda & 12.35 & 22 & & & 3.86 & 84 \\
\hline Echinodermata & 1.51 & 10 & & & 6.16 & 134 \\
\hline Diversos & & & & & 3.49 & 76 \\
\hline & & & & & & \\
\hline
\end{tabular}

\begin{tabular}{|c|c|c|c|c|c|c|}
\hline ESTAÇ̃ES & \multicolumn{2}{|c|}{ B 09 } & \multicolumn{2}{c|}{ B 10} & \multicolumn{2}{c|}{ B 11 } \\
\hline PROFUNDIDADE (m) & \multicolumn{2}{|c|}{110} & \multicolumn{2}{c|}{49} & \multicolumn{2}{c|}{10} \\
\hline PERCENT. DENS. & $\%$ & ind. $/ \mathrm{m}^{2}$ & $\%$ & ind. $/ \mathrm{m}^{2}$ & $\%$ & ind. $/ \mathrm{m}^{2}$ \\
\hline Polychaeta & 20.69 & 452 & 85.03 & 216 & 10.24 & 126 \\
\hline Crustacea & 7.14 & 156 & 8.66 & 22 & 12.68 & 156 \\
\hline Mollusca & 67.76 & 1480 & & & 0.65 & 800 \\
\hline Nematoda & 2.38 & 52 & 3.93 & 10 & 2.60 & 32 \\
\hline Echinodermata & 1.64 & 36 & & & & \\
\hline Diversos & 0.36 & 8 & 2.36 & 6 & 9.43 & 116 \\
\hline & & & & & & \\
\hline
\end{tabular}




\begin{tabular}{|l|c|c|c|c|c|c|}
\hline ESTAÇÕES & \multicolumn{2}{|c|}{ B 12} & \multicolumn{2}{c|}{ B 13 } & \multicolumn{2}{c|}{ B. 14} \\
\hline PROFUNDIDADE (m) & \multicolumn{2}{|c|}{36} & \multicolumn{2}{c|}{72} & \multicolumn{2}{c|}{103} \\
\hline PERCENT. DENS. & $\%$ & ind. $/ \mathrm{m}^{2}$ & $\%$ & ind. $/ \mathrm{m}^{2}$ & $\%$ & ind. $/ \mathrm{m}^{2}$ \\
\hline Polychaeta & 100.00 & 16 & 15.71 & 176 & 100.00 & 72 \\
\hline Crustacea & & & 1.25 & 14 & & \\
\hline Mollusca & & & 82.32 & 922 & & \\
\hline Nematoda & & & & & & \\
\hline Echinodermata & & & 0.71 & 8 & & \\
\hline Diverses & & & & & & \\
\hline & & & & & & \\
\hline
\end{tabular}

\begin{tabular}{|l|r|r|r|r|r|r|}
\hline ESTAÇÕES & \multicolumn{2}{|c|}{ B 15} & \multicolumn{2}{c|}{ B 16} & \multicolumn{2}{c|}{ B 17 } \\
\hline PROFUndIDADE (m) & \multicolumn{2}{|c|}{120} & \multicolumn{2}{c|}{166} & \multicolumn{2}{|c|}{263} \\
\hline PERCENT. DENS. & $\%$ & ind. $/ \mathrm{m}^{2}$ & $\%$ & ind. $/ \mathrm{m}^{2}$ & $\%$ & ind. $/ \mathrm{m}^{2}$ \\
\hline Polychaeta & 14.92 & 120 & 55.73 & 136 & & \\
\hline Crustacea & 7.21 & 58 & 0.81 & 2 & & \\
\hline Mollusca & 29.10 & 234 & 13.11 & 32 & & \\
\hline Nematoda & 7.21 & 58 & 8.19 & 20 & & \\
\hline Echinodermata & 3.48 & 28 & 4.09 & 10 & & \\
\hline Diversos & 38.05 & 306 & 18.03 & 44 & & \\
\hline & & & & & & \\
\hline
\end{tabular}

\begin{tabular}{|c|r|r|r|r|r|r|}
\hline ESTAÇÕES & \multicolumn{2}{|c|}{ C 18} & \multicolumn{2}{c|}{ C 19} & \multicolumn{2}{c|}{ C 20 } \\
\hline PROFUNDIDADE (m) & \multicolumn{2}{|c|}{21} & \multicolumn{2}{c|}{67} & \multicolumn{2}{c|}{111} \\
\hline PERCENT. DENS. & $\%$ & ind. $/ \mathrm{m}^{2}$ & \multicolumn{1}{c|}{$\%$} & ind. $/ \mathrm{m}^{2}$ & \multicolumn{1}{c|}{$\%$} & ind. $/ \mathrm{m}^{2}$ \\
\hline Pelychaeta & 100.00 & 16 & 25.20 & 1.190 & 89.94 & 912 \\
\hline Crustacea & & & 1.44 & 18 & 8.08 & 82 \\
\hline Mollusca & & & & & & \\
\hline Nematoda & & & 1.44 & 18 & 0.19 & 2 \\
\hline Echinodermata & & & 1.12 & 14 & 1.27 & 18 \\
\hline Diversos & & & 0.80 & 10 & & \\
\hline & & & & & & \\
\hline
\end{tabular}




\begin{tabular}{|l|c|c|c|c|c|c|}
\hline \multicolumn{1}{|c|}{ ESTAÇÕES } & \multicolumn{2}{|c|}{ C 21} & \multicolumn{2}{c|}{ C 22 } & \multicolumn{2}{c|}{ C 23 } \\
\hline PROFUNDIDADE (m) & \multicolumn{2}{|c|}{110} & \multicolumn{2}{c|}{146} & \multicolumn{2}{c|}{155} \\
\hline PE RCENT. DENS. & $\%$ & ind. $/ \mathrm{m}^{2}$ & $\%$ & ind. $/ \mathrm{m}^{2}$ & $\%$ & ind./m \\
\hline Polychaeta & 100.00 & 114 & & & 72.41 & 42 \\
\hline Crustacea & & & & & & \\
\hline Mollusca & & & & & & \\
\hline Nematoda & & & & & 24.13 & 14 \\
\hline Echinodermata & & & & & 3.44 & 2 \\
\hline Diversos & & & & & & \\
\hline & & & & & & \\
\hline
\end{tabular}

\begin{tabular}{|l|c|c|c|c|c|c|}
\hline \multicolumn{1}{|c|}{ ESTACÕES } & \multicolumn{2}{|c|}{ C 25 } & \multicolumn{2}{c|}{ C 27 } & \multicolumn{2}{c|}{ C 28 } \\
\hline PROFUNDIDADE (m) & \multicolumn{2}{|c|}{255} & \multicolumn{2}{c|}{165} & \multicolumn{2}{c|}{105} \\
\hline PERCENT. DENS. & $\%$ & ind. $/ \mathrm{m}^{2}$ & $\%$ & ind. $/ \mathrm{m}^{2}$ & $\%$ & ind. $/ \mathrm{m}^{2}$ \\
\hline Polychaeta & 58.33 & 28 & 100.00 & 24 & & \\
\hline Crustacea & & & & & & \\
\hline Mollusca & & & & & & \\
\hline Nematoda & & & & & & \\
\hline Echinodermata & & & & & & \\
\hline Diversos & 41.66 & 20 & 100.00 & 2 & & \\
\hline & & & & & & \\
\hline
\end{tabular}

\begin{tabular}{|l|c|c|c|c|c|c|}
\hline \multicolumn{1}{|c|}{ BSTAÇÕES } & \multicolumn{2}{|c|}{ C 29} & \multicolumn{2}{c|}{ D 30 } & \multicolumn{2}{c|}{ D 31 } \\
\hline PROFUNDIDADE (m) & \multicolumn{2}{|c|}{25} & $34-35$ & \multicolumn{2}{c|}{63} \\
\hline PERCENT. DENS. & $\%$ & ind. $/ \mathrm{m}^{2}$ & $\%$ & ind. $/ \mathrm{m}^{2}$ & $\%$ & ind. $/ \mathrm{m}^{2}$ \\
\hline Polychaeta & 69.91 & 688 & & & 20.17 & 232 \\
\hline Crustacea & 20.73 & 204 & & & 9.04 & 104 \\
\hline Mollusca & 2.43 & 24 & & & 33.56 & 386 \\
\hline Nematoda & 3.86 & 38 & & & & \\
\hline Bchinodermata & 1.01 & 10 & & & 11.47 & 132 \\
\hline Diversos & 2.03 & 20 & & & 25.73 & 296 \\
\hline & & & & & & \\
\hline
\end{tabular}




\begin{tabular}{|c|c|c|c|c|c|c|}
\hline ESTAÇÕES & \multicolumn{2}{|c|}{ D 32 } & \multicolumn{2}{c|}{ D 33 } & \multicolumn{2}{c|}{ D 34 } \\
\hline PROFUNDIDADE (m) & \multicolumn{2}{|c|}{102} & \multicolumn{2}{c|}{162} & \multicolumn{2}{c|}{185} \\
\hline PERCENT. DENS. & $\%$ & ind. $/ \mathrm{m}^{2}$ & $\%$ & ind. $/ \mathrm{m}^{2}$ & $\%$ & ind. $/ \mathrm{m}^{2}$ \\
\hline Polychaeta & & & & & 14.87 & 72 \\
\hline Crustacea & & & & & 8.67 & 42 \\
\hline Moliusca & & & & & 30.57 & 148 \\
\hline Nematoda & & & & & 30.29 & 150 \\
\hline Echinodermata & & & & & 2.06 & 10 \\
\hline Diversos & 100.00 & 4 & & & 12.80 & 62 \\
\hline & & & & & & \\
\hline
\end{tabular}

\begin{tabular}{|l|r|r|r|r|r|r|}
\hline ESTAÇÕES & \multicolumn{2}{|c|}{ D 35} & \multicolumn{2}{c|}{ D 36 } & \multicolumn{2}{c|}{ D 37 } \\
\hline PROFUNDIDADE (m) & \multicolumn{2}{|c|}{191} & \multicolumn{2}{c|}{245} & \multicolumn{2}{c|}{301} \\
\hline PERCENT. DENS. & $\%$ & ind. $/ \mathrm{m}^{2}$ & \multicolumn{1}{c|}{$\%$} & ind. $/ \mathrm{m}^{2}$ & \multicolumn{1}{c|}{$\%$} & ind. $\mathrm{m}^{2}$ \\
\hline Polychaeta & 100.00 & 16 & 25.33 & 152 & 17.47 & 36 \\
\hline Crustacea & & & 10.66 & 64 & 21.35 & 44 \\
\hline Mollusca & & & 22.33 & 134 & 8.73 & 13 \\
\hline Nematoda & & & 7.00 & 42 & 3.88 & 8 \\
\hline Echinodermata & & & 18.00 & 108 & 34.95 & 72 \\
\hline Diversos & & & 16.00 & 100 & 13.59 & 28 \\
\hline & & & & & & \\
\hline
\end{tabular}

\begin{tabular}{|l|r|r|r|r|r|r|}
\hline ESTAÇÕES & \multicolumn{2}{|c|}{ E 39 } & \multicolumn{2}{c|}{ E 40 } & \multicolumn{2}{c|}{ E 42 } \\
\hline PROFUNDIDADE (m) & \multicolumn{2}{|c|}{60} & \multicolumn{2}{c|}{181} & \multicolumn{2}{c|}{140} \\
\hline PERCENT. DENS. & \multicolumn{1}{c|}{$\%$} & ind. $/ \mathrm{m}^{2}$ & \multicolumn{1}{c|}{$\%$} & ind. $/ \mathrm{m}^{2}$ & $\%$ & ind. $/ \mathrm{m}^{2}$ \\
\hline Polychaeta & 84.27 & 2530 & 92.09 & 722 & 84.95 & 1626 \\
\hline Crustacea & 5.79 & 174 & 2.80 & 22 & 1.04 & 20 \\
\hline Mollusca & 2.46 & 74 & 1.78 & 14 & 4.59 & 88 \\
\hline Nematoda & 6.12 & 184 & 2.29 & 18 & 6.37 & 122 \\
\hline Echinodermata & 0.73 & 22 & & & 0.20 & 4 \\
\hline Diversos & 0.59 & 18 & 1.02 & 8 & 2.82 & 54 \\
\hline & & & & & & \\
\hline
\end{tabular}




\begin{tabular}{|l|c|c|c|c|c|c|}
\hline \multicolumn{1}{|c|}{ ESTACÕES } & \multicolumn{2}{|c|}{ E 43} & \multicolumn{2}{|c|}{ E 46 } & \multicolumn{2}{|c|}{ E 47 } \\
\hline PROFUNDIDADE (m) & \multicolumn{2}{|c|}{138} & \multicolumn{2}{|c|}{46} & \multicolumn{2}{|c|}{47} \\
\hline PERCENT. DENS. & $\%$ & ind. $/ \mathrm{m}^{2}$ & $\%$ & ind. $/ \mathrm{m}^{2}$ & $\%$ & ind. $/ \mathrm{m}^{2}$ \\
\hline Polychaeta & & & & & 88.23 & 30 \\
\hline Crustacea & & & & & & \\
\hline Mollusca & & & & & & \\
\hline Nematoda & & & & & & \\
\hline Echinodermata & & & & & & \\
\hline Diversos & & & & & 11.76 & 4 \\
\hline & & & & & & \\
\hline
\end{tabular}

\begin{tabular}{|l|c|c|c|c|c|c|}
\hline \multicolumn{1}{|c|}{ ESTAÇÕES } & \multicolumn{2}{|c|}{ F 49 } & \multicolumn{2}{c|}{ F 50 } & \multicolumn{2}{c|}{ F 51 } \\
\hline PROFUNDIDADE (m) & \multicolumn{2}{|c|}{42} & \multicolumn{2}{c|}{70} & \multicolumn{2}{c|}{95} \\
\hline PERCENT, DEND. & $\%$ & ind. $/ \mathrm{m}^{2}$ & $\%$ & ind./m & $\%$ & ind. $/ \mathrm{m}^{2}$ \\
\hline Polychaeta & 100.00 & 14 & 34.46 & 1092 & 76.19 & 32 \\
\hline Crustacea & & & 13.57 & 430 & & \\
\hline Mollusca & & & 20.89 & 662 & & \\
\hline Nematoda & & & 10.73 & 340 & 4.76 & 2 \\
\hline Echinodermata & & & 3.59 & 114 & 19.04 & 8 \\
\hline Diversos & & & 16.72 & 530 & & \\
\hline & & & & & & \\
\hline
\end{tabular}

\begin{tabular}{|l|c|c|c|c|c|c|}
\hline ESTAÇÕES & \multicolumn{2}{|c|}{ F 52 } & \multicolumn{2}{c|}{ F 53 } & \multicolumn{2}{c|}{ F 54 } \\
\hline PROFUNDIDADE (m) & \multicolumn{2}{|c|}{125} & \multicolumn{2}{c|}{155} & \multicolumn{2}{c|}{190} \\
\hline PERCENT. DENS. & $\%$ & ind. $/ \mathrm{m}^{2}$ & $\%$ & ind. $/ \mathrm{m}^{2}$ & \multicolumn{1}{c|}{$\%$} & ind. $/ \mathrm{m}^{2}$ \\
\hline Polychaeta & 48.00 & 240 & 42.18 & 54 & 23.35 & 446 \\
\hline Crustacea & 11.60 & 58 & 6.25 & 8 & 19.09 & 156 \\
\hline Mollusca & 13.60 & 68 & 12.50 & 16 & 47.74 & 1460 \\
\hline Nematoda & 5.20 & 26 & 10.93 & 14 & 1.76 & 54 \\
\hline Echinodermata & 10.40 & 52 & 0.98 & & 0.22 & 30 \\
\hline Diversos & 11.20 & 56 & 28.12 & 36 & 15.82 & 484 \\
\hline & & & & & & \\
\hline
\end{tabular}




\begin{tabular}{|l|c|c|c|c|c|c|}
\hline ESTAÇÕES & \multicolumn{2}{|c|}{ F 55 } & \multicolumn{2}{c|}{ F 56 } & \multicolumn{2}{c|}{ F 59 } \\
\hline PROFUNDIDADE (m) & \multicolumn{2}{|c|}{262} & \multicolumn{2}{c|}{$390-393$} & \multicolumn{2}{c|}{104} \\
\hline PERCENT. DENS. & $\%$ & ind. $/ \mathrm{m}^{2}$ & $\%$ & ind. $/ \mathrm{m}^{2}$ & $\%$ & ind. $/ \mathrm{m}^{2}$ \\
\hline Polychaeta & 23.35 & 206 & & & 16.45 & 1316 \\
\hline Crustacea & 17.68 & 156 & & & 8.77 & 702 \\
\hline Mollusca & 51.47 & 454 & & & 37.04 & 2962 \\
\hline Nematoda & 0.22 & 2 & & & 4.45 & 356 \\
\hline Echinodermata & 0.22 & 2 & & & 19.48 & 1558 \\
\hline Diversos & 7.02 & 62 & & & 13.78 & 1102 \\
\hline & & & & & & \\
\hline
\end{tabular}

\begin{tabular}{|l|r|r|r|r|r|c|}
\hline \multicolumn{1}{|c|}{ ESTAÇÕES } & \multicolumn{2}{c|}{ G 60 } & \multicolumn{2}{c|}{ G 61 } & \multicolumn{2}{c|}{ G 62 } \\
\hline PROFUNDIDADE (m) & \multicolumn{2}{|c|}{18} & \multicolumn{2}{c|}{51} & \multicolumn{2}{c|}{75} \\
\hline PERCENT. DENS. & \multicolumn{1}{c|}{$\%$} & ind. $/ \mathrm{m}^{2}$ & \multicolumn{1}{c|}{$\%$} & ind. $/ \mathrm{m}^{2}$ & \multicolumn{1}{c|}{$\%$} & ind. $\mathrm{m}^{2}$ \\
\hline Polychaeta & 62.46 & 476 & 20.78 & 960 & 50.00 & 16 \\
\hline Crustacea & 11.02 & 84 & 31.00 & 1432 & & \\
\hline Mollusca & & & 3.85 & 178 & & \\
\hline Nematoda & 2.36 & 18 & 30.87 & 1426 & 31.25 & 10 \\
\hline Echinodermata & 1.04 & 8 & 12.64 & 584 & 18.75 & 6 \\
\hline Diversos & 23.09 & 176 & 0.82 & 38 & & \\
\hline & & & & & & \\
\hline
\end{tabular}

\begin{tabular}{|l|r|r|r|r|r|r|}
\hline ESTAÇÕES & \multicolumn{2}{|c|}{ G 63 } & \multicolumn{2}{c|}{ G 64 } & \multicolumn{2}{c|}{ G 65 } \\
\hline PROFUndIDADE $(\mathrm{m})$ & \multicolumn{2}{|c|}{335} & \multicolumn{2}{c|}{140} & \multicolumn{2}{c|}{177} \\
\hline PERCENT. DENS. & $\%$ & ind. $/ \mathrm{m}^{2}$ & \multicolumn{1}{c|}{$\%$} & ind. $/ \mathrm{m}^{2}$ & \multicolumn{1}{c|}{$\%$} & ind. $/ \mathrm{m}^{2}$ \\
\hline Polychaeta & & & 41.42 & 522 & 90.32 & 56 \\
\hline Crustacea & & & 9.52 & 120 & 9.67 & 6 \\
\hline Mollusca & & & 3.33 & 42 & & \\
\hline Nematoda & & & 30.47 & 384 & & \\
\hline Echinodermata & & & 5.55 & 70 & & \\
\hline Diversos & & & 9.68 & 122 & & \\
\hline & & & & & & \\
\hline
\end{tabular}




\begin{tabular}{|l|c|c|c|c|c|c|}
\hline ESTAÇÕES & \multicolumn{2}{|c|}{ G 66 } & \multicolumn{2}{c|}{ G 68 } & \multicolumn{2}{c|}{ G 69 } \\
\hline PROFUndidADE (m) & \multicolumn{2}{|c|}{200} & \multicolumn{2}{c|}{394} & \multicolumn{2}{c|}{200} \\
\hline PERCENT. DENS. & $\%$ & ind. $/ \mathrm{m}^{2}$ & $\%$ & ind. $/ \mathrm{m}^{2}$ & $\%$ & ind. $/ \mathrm{m}^{2}$ \\
\hline Polychaeta & 89.47 & 34 & & & & \\
\hline Crustacea & & & & & & \\
\hline Mollus ca & & & & & & \\
\hline Nematoda & 10.52 & 4 & & & & \\
\hline Echinodermata & & & & & & \\
\hline Diversos & & & & & & \\
\hline & & & & & & \\
\hline
\end{tabular}

\begin{tabular}{|l|r|r|r|r|r|r|}
\hline \multicolumn{1}{|c|}{ ESTAÇÕES } & \multicolumn{2}{|c|}{ G 73 } & \multicolumn{2}{c|}{ G 74 } & \multicolumn{2}{c|}{ G 75 } \\
\hline PROFUNDIDADE $(\mathrm{m})$ & \multicolumn{2}{|c|}{35} & \multicolumn{2}{c|}{30} & \multicolumn{2}{c|}{$185-207$} \\
\hline PERCENT. DENS. & $\%$ & ind. $/ \mathrm{m}^{2}$ & $\%$ & ind. $/ \mathrm{m}^{2}$ & \multicolumn{1}{c|}{$\%$} & ind./m \\
\hline Polychaeta & 45.94 & 68 & 41.17 & 154 & 29.63 & 614 \\
\hline Crustacea & 25.67 & 38 & 40.64 & 152 & & \\
\hline Mollusca & 2.70 & 4 & & & 11.38 & 236 \\
\hline Nematoda & 1.35 & 2 & 13.90 & 52 & 1.25 & 26 \\
\hline E.chinodermata & & & 4.27 & 16 & 0.19 & 4 \\
\hline Diversos & 24.32 & 36 & & & 57.52 & 1192 \\
\hline & & & & & & \\
\hline
\end{tabular}

\begin{tabular}{|l|c|c|c|c|c|c|}
\hline \multicolumn{1}{|c|}{ ESTAÇÕES } & \multicolumn{2}{|c|}{ G 77 } & \multicolumn{2}{c|}{ G 78 } & \multicolumn{2}{c|}{ G 79 } \\
\hline PROFUNDIDADE (m) & \multicolumn{2}{|c|}{190} & \multicolumn{2}{c|}{$46-54$} & \multicolumn{2}{c|}{37} \\
\hline PERCENT. DENS. & $\%$ & ind. $/ \mathrm{m}^{2}$ & $\%$ & ind. $/ \mathrm{m}^{2}$ & $\%$ & ind. $/ \mathrm{m}^{2}$ \\
\hline Polychaeta & 20.91 & 220 & & & 100.00 & 144 \\
\hline Crustacea & 61.78 & 650 & & & & \\
\hline Mollusca & 13.68 & 144 & & & & \\
\hline Nematoda & 0.95 & 10 & & & & \\
\hline Echinodermata & 1.52 & 16 & & & & \\
\hline Diversos & 1.14 & 12 & & & & \\
\hline & & & & & & \\
\hline
\end{tabular}




\begin{tabular}{|l|r|r|r|r|r|r|}
\hline \multicolumn{1}{|c|}{ ESTAÇÕES } & \multicolumn{2}{|c|}{ G.80 } & \multicolumn{2}{c|}{ G 81} & \multicolumn{2}{c|}{ H 83} \\
\hline PROFUndIDADE (m) & \multicolumn{2}{|c|}{136} & \multicolumn{2}{c|}{233} & \multicolumn{2}{c|}{.24} \\
\hline PERCENT. DENS. & $\%$ & ind. $/ \mathrm{m}^{2}$ & \multicolumn{1}{c|}{$\%$} & ind. $/ \mathrm{m}^{2}$ & \multicolumn{1}{c|}{$\%$} & ind. $/ \mathrm{m}^{2}$ \\
\hline Polychaeta & 65.06 & & 42.25 & & 1.23 & \\
\hline Crustacea & 6.16 & & 7.01 & & 7.86 & \\
\hline Mollus ca & 17.17 & & 0.18 & & 87.84 & \\
\hline Nematoda & 3.42 & & 41.32 & & 0.30 & \\
\hline Echinodermata & & & 0.92 & & 0.46 & \\
\hline Diversos & 6.16 & & 8.30 & & 2.28 & \\
\hline & & & & & & \\
\hline
\end{tabular}

\begin{tabular}{|l|r|r|r|r|r|r|}
\hline \multicolumn{1}{|c|}{ ESTAÇÕES } & \multicolumn{2}{|c|}{ H 84} & \multicolumn{2}{c|}{ H 85} & \multicolumn{2}{c|}{ H 86} \\
\hline PROFUNDIDADE (m) & \multicolumn{2}{|c|}{50} & \multicolumn{2}{c|}{51} & \multicolumn{2}{c|}{95} \\
\hline PERCENT. DENS. & \multicolumn{1}{|c|}{$\%$} & ind. $/ \mathrm{m}^{2}$ & \multicolumn{1}{c|}{$\%$} & ind. $/ \mathrm{m}^{2}$ & \multicolumn{1}{c|}{$\%$} & ind./m \\
\hline Polychaeta & 13.66 & 96 & 58.61 & 182 & 69.56 & 2422 \\
\hline Crustacea & 18.46 & 612 & 10.11 & 246 & 12.40 & 418 \\
\hline Mollusca & 36.33 & 484 & 1.30 & 54 & 0.49 & 14 \\
\hline Nematoda & 9.60 & 128 & 8.18 & 338 & 14.82 & 416 \\
\hline Echinode rmata & 2.40 & 32 & 0.33 & 14 & & \\
\hline Diversos & 19.51 & 260 & 21.44 & 886 & 2.70 & 76 \\
\hline & & & & & & \\
\hline
\end{tabular}

\begin{tabular}{|l|r|r|r|r|r|r|}
\hline ESTAÇÕES & \multicolumn{2}{|c|}{ H 87} & \multicolumn{2}{c|}{ H 89} & \multicolumn{2}{c|}{ H 90 } \\
\hline PROFUNDIDADE (m) & \multicolumn{2}{|c|}{105} & \multicolumn{2}{c|}{105} & \multicolumn{2}{c|}{128} \\
\hline PERCENT. DENS. & \multicolumn{1}{|c|}{$\%$} & ind. $/ \mathrm{m}^{2}$ & \multicolumn{1}{c|}{$\%$} & ind. $/ \mathrm{m}^{2}$ & \multicolumn{1}{c|}{$\%$} & ind. $/ \mathrm{m}^{2}$ \\
\hline Polychaeta & 21.40 & 274 & 73.32 & 5646 & 47.33 & 870 \\
\hline Crustacea & 0.46 & 6 & 1.63 & 126 & 12.29 & 226 \\
\hline Mollusca & 6.25 & 80 & 0.25 & 20 & 7.18 & 132 \\
\hline Nematoda & 60.46 & 774 & 24.20 & 1864 & 14.79 & 272 \\
\hline Echinodermata & & & 0.02 & 2 & 5.98 & 110 \\
\hline Diversos & 11.40 & 146 & 0.54 & 42 & 12.40 & 228 \\
\hline & & & & & & \\
\hline
\end{tabular}




\begin{tabular}{|l|r|r|r|r|r|c|}
\hline \multicolumn{1}{|c|}{ ESTAÇÕES } & \multicolumn{2}{|c|}{ H.91 } & \multicolumn{2}{c|}{ H 92} & \multicolumn{2}{c|}{ H 94} \\
\hline PROFUNDIDADE (m) & \multicolumn{2}{|c|}{151} & \multicolumn{2}{c|}{164} & \multicolumn{2}{c|}{170} \\
\hline PERCENT. DENS. & \multicolumn{1}{|c|}{$\%$} & ind. $/ \mathrm{m}^{2}$ & $\%$ & ind. $/ \mathrm{m}^{2}$ & $\%$ & ind. $/ \mathrm{m}^{2}$ \\
\hline Polychaeta & 32.15 & 236 & 44.07 & 878 & 100.00 & 34 \\
\hline Crustacea & 26.70 & 196 & 43.17 & 860 & & \\
\hline Mollusca & 4.90 & 36 & 2.61 & 52 & & \\
\hline Nemacoda & 22.61 & 166 & 8.63 & 172 & & \\
\hline Echinodermata & 0.81 & 6 & 0.30 & 6 & & \\
\hline Diversos & 12.80 & 94 & 1.20 & 24 & & \\
\hline & & & & & & \\
\hline
\end{tabular}

\begin{tabular}{|l|c|c|c|c|c|c|}
\hline \multicolumn{1}{|c|}{ ESTAÇÕES } & \multicolumn{2}{|c|}{ H 95 } & \multicolumn{2}{c|}{ H 97 } & \multicolumn{2}{c|}{ I 99 } \\
\hline PROFUNDIDADE (m) & \multicolumn{2}{|c|}{195} & \multicolumn{2}{c|}{1017} & \multicolumn{2}{c|}{44} \\
\hline PERCENT. DENS. & $\%$ & ind. $/ \mathrm{m}^{2}$ & $\%$ & ind. $/ \mathrm{m}^{2}$ & $\%$ & ind. $/ \mathrm{m}^{2}$ \\
\hline Polychaeta & 67.50 & 54 & 55.10 & 162 & 7.96 & 188 \\
\hline Crustacea & 20.00 & 16 & 12.92 & 38 & 22.37 & 528 \\
\hline Mollusca & & & 10.88 & 32 & 52.96 & 1250 \\
\hline Nematoda & 5.00 & 4 & 9.52 & 28 & 3.05 & 72 \\
\hline Echinodermata & & & 4.08 & 12 & 4.74 & 112 \\
\hline Diversos & 7.50 & 6 & 7.48 & 22 & 8.89 & 210 \\
\hline & & & & & & \\
\hline
\end{tabular}

\begin{tabular}{|l|r|r|r|r|r|r|}
\hline \multicolumn{1}{|c|}{ ESTAÇÕES } & \multicolumn{2}{|c|}{ I 100} & \multicolumn{2}{c|}{ I 101 } & \multicolumn{2}{c|}{ I 102 } \\
\hline PROFUnDIDADE $(\mathrm{m})$ & \multicolumn{2}{|c|}{74} & \multicolumn{2}{c|}{84} & \multicolumn{2}{c|}{95} \\
\hline PERCENT. DENS. & \multicolumn{1}{c|}{$\%$} & ind. $/ \mathrm{m}^{2}$ & \multicolumn{1}{c|}{$\%$} & ind. $/ \mathrm{m}^{2}$ & $\%$ & ind. $/ \mathrm{m}^{2}$ \\
\hline Polychaeta & 46.41 & 1464 & 66.19 & 1132 & 37.46 & 236 \\
\hline Crust acea & 6.27 & 198 & 7.36 & 126 & 3.17 & 20 \\
\hline Mollusca & 0.57 & 18 & 3.50 & 60 & 33.33 & 210 \\
\hline Nematoda & 16.48 & 520 & 17.19 & 294 & 7.30 & 46 \\
\hline Echinodermata & 1.83 & 58 & 2.10 & 36 & 1.90 & 12 \\
\hline Diversos & 28.40 & 896 & 3.62 & 62 & 16.82 & 106 \\
\hline & & & & & & \\
\hline
\end{tabular}




\begin{tabular}{|l|r|l|r|r|r|r|}
\hline ESTAÇÕES & \multicolumn{2}{|c|}{ I 103} & \multicolumn{2}{c|}{ I 104 } & \multicolumn{2}{c|}{ I 105 } \\
\hline PROFUNDIDADE (m) & \multicolumn{2}{|c|}{132} & \multicolumn{2}{c|}{$507-562$} & \multicolumn{2}{c|}{866} \\
\hline PERCENT, DENS. & $\%$ & ind. $/ \mathrm{m}^{2}$ & $\%$ & ind. $/ \mathrm{m}^{2}$ & $\%$ & ind. $/ \mathrm{m}^{2}$ \\
\hline Polychaeta & 100.00 & & 28.94 & & & \\
\hline Crustacea & & & 10.52 & & & \\
\hline Mollusca & & & 26.31 & & & \\
\hline Nematoda & & & 10.52 & & & \\
\hline Echinodermata & & & 5.26 & & & \\
\hline Diversos & & & 18.42 & & & \\
\hline & & & & & & \\
\hline
\end{tabular}

\begin{tabular}{|l|r|r|r|r|r|c|}
\hline \multicolumn{1}{|c|}{ ESTAC̄̃ES } & \multicolumn{2}{c|}{ I 109} & \multicolumn{2}{c|}{ I 110} & \multicolumn{2}{c|}{ J 112} \\
\hline PROFUNDIDADE (m) & \multicolumn{2}{|c|}{33} & \multicolumn{2}{c|}{22} & \multicolumn{2}{c|}{104} \\
\hline PERCENT. DENS. & \multicolumn{1}{c|}{$\%$} & ind. $/ \mathrm{m}^{2}$ & \multicolumn{1}{c|}{$\%$} & ind. $/ \mathrm{m}^{2}$ & \multicolumn{1}{c|}{$\%$} & ind. $/ \mathrm{m}^{2}$ \\
\hline Polychaeta & 85.41 & 82 & 3.12 & 244 & 15.23 & 128 \\
\hline Crustacea & 8.33 & 8 & 59.63 & 2 & 0.23 & \\
\hline Mollusca & & & 31.41 & 2450 & 2.85 & 24 \\
\hline Nematoda & 6.25 & 6 & 1.69 & 132 & 3.57 & 30 \\
\hline Echinodermata & & & 2.41 & 188 & & \\
\hline Diversos & & & 1.71 & 134 & 78.57 & 660 \\
\hline & & & & & & \\
\hline
\end{tabular}

\begin{tabular}{|l|r|r|r|r|r|r|}
\hline \multicolumn{1}{|c|}{ ESTAÇÕES } & \multicolumn{2}{|c|}{$\mathrm{J} 113$} & \multicolumn{2}{c|}{$\mathrm{J} 114$} & \multicolumn{2}{c|}{$\mathrm{J} 115$} \\
\hline PROFUNDIDADE (m) & \multicolumn{2}{|c|}{147} & \multicolumn{2}{c|}{168} & \multicolumn{2}{c|}{234} \\
\hline PERCENT. DENS. & \multicolumn{1}{c|}{$\%$} & ind. $/ \mathrm{m}^{2}$ & \multicolumn{1}{c|}{$\%$} & \multicolumn{1}{c|}{ ind. $/ \mathrm{m}^{2}$} & \multicolumn{1}{c|}{$\%$} & ind. $/ \mathrm{m}^{2}$ \\
\hline Polychaeta & 33.33 & 38 & 2.35 & 836 & 7.50 & 56 \\
\hline Crustace a & & & 1.47 & 522 & 1.60 & 12 \\
\hline Mollusca & 57.89 & 66 & 48.01 & 17030 & 79.08 & 590 \\
\hline Nematoda & 8.77 & 10 & 0.21 & 78 & 2.94 & 22 \\
\hline Echinodermata & & & 0.11 & 42 & 0.26 & 2 \\
\hline Diversos & & & 47.81 & 16960 & 8.57 & 64 \\
\hline & & & & & & \\
\hline
\end{tabular}


Mediram-se as salinidades por intermédio de um salinômetro Beckman R57B, permitindo uma precisão, dentro das condições de medidas em Campanha, de aproximadamente $0,01 \%$ oo.

Após coleta de água em garrafa BOD de $300 \mathrm{ml}$ e adição imediata de reativos a titulação de oxigênio foi feita pelo método de Winkler (David, Murail \& Panouse, 1977).

Os sedimentos recolhidos foram lavados imediatamente com água do mar, no próprio navio, através de tamis com malha de $1 \mathrm{~mm}$. A sobra do tamis foi fixada em formol neutro a $10 \%$.

\section{RESULTADOS}

No plano quantitativo, observamos a presença constante de 5 grupos: Polychaeta, Crustacea, Mollusca, Nematoda, Echinodermata, sendo dentre eles os menos freqüentes Nematoda e Echinodermata.

A presença desses grupos em Kerguelen já foi assinalada por: Chardy, Desbruyères \& Laurec, 1976; de Bovée, 1976; Desbruyères \& Guille, 1973, Rullier, 1966; Fauvel, 1953; Guille \& Soyer, 1976; Arnaud, $1974 .$.

O grupo dos Polychaeta é o que apresenta maior número de espécimes, $24 \%$, seguido do dos Crustacea, $23 \%$.

O coeficiente de afinidade entre os dois grupos mais representados é da ordem de $74 \%$. A análise dos resultados quantitativos mostra que as maiores densidades registradas são igualmente grandes, estando compreendidas entre 5.646 ind $/ \mathrm{m}^{2}$ e 18.542 ind $/ \mathrm{m}^{2}$ (Fig. 3).

Tomando como referência a radial E, verificamos que o grupo dos Polychaeta representa $86 \%$ da dominância, seguido por Nematoda $6 \%$, Crustacea $4 \%$, Mollusca $3 \%$, Echinodermata $0,5 \%$ e diversos $1 \%$.

Um trabalho análogo foi feito por De Bovée \& Soyer, 1977 na plataforma continental de Kerguelen, no que concerne ao meiobentos.

As densidades registradas por estes autores mostram grande variabilidade, indo de 61 ind $/ 10 \mathrm{~cm}^{2}$ (E 40) e 3.193 ind $/ 10 \mathrm{~cm}^{2}$ (G 81), com um valor médio de 907 ind $/ 10 \mathrm{~cm}^{2}$, sendo a relação entre densidade máxima e mínima de 1 a 52 e indicativa da grande heterogeneidade dos biótopos encontrados.

Nosso trabalho, estudando a mesma região, com material proveniente da mesma época do ano, em idênticas condições ambientais, no que diz respeito ao macroben-, tos, apresentou os seguintes resultados:

A maior densidade de especimens é encontrada a uma profundidade de $1,68 \mathrm{~m}$; salinidade entre $33,773 \%$ oo e $33,382^{\circ} / 00$; temperatura entre $4,01^{\circ} \mathrm{C}$ e $4,84^{\circ} \mathrm{C}$ num substrato arenoso acinzentado com Bryozoa. Uma diminuição sensível processa-se na profundidade de $346 \mathrm{~m}$; salinidade entre $33,799 \%$ oo e 34,802\% $/ 00$; temperatura entre $1,9^{\circ} \mathrm{C}$ e $4,60^{\circ} \mathrm{C}$ e substrato de blocos de basalto.

A densidade diminui nos substratos entre 76 e 90 ind $/ \mathrm{cm}^{2}$, com aumento de profundidade e o afastamento da costa, tal como verificaram De Bovée \& Soyer, 1977 em meiobentos.

No meiobentos, como no macrobentos, os Annelida Polychaeta são os táxons mais numerosos e mais freqüentemente representados.

$\mathrm{Na}$ areia, no conjunto das estações, as densidades estão compreendidas entre 16 ind $/ \mathrm{m}^{2}$ e 958 ind $/ \mathrm{m}^{2} ; \mathrm{e}$, na vasa, entre 16 ind $/ \mathrm{m}^{2}$ e $5.646 \mathrm{ind} / \mathrm{m}^{2}$. 


\section{DISCUSSÃO}

Duas grandes tendências orientam as pesquisas no que concerne ao macrobento da região Subantártica, no plano quantitativo:

1) As interaçбes recíprocas entre a fauna Anelidiana e o substrato móvel e o conjunto do macrobentos, que nos levam a pensar numa relação quase fisiológica entre os sedimentos, as populações e os organismos. Com efeito, os sedimentos marinhos podem ser considerados, segundo Cahet, 1974, como um organismo dinâmico essencialmente heterotrófico. "Do ponto de vista biogeoquímico, os sedimentos realizam funções importantes na economia marinha e aí os microrganismos fazem o liame entre a matéria inerte e a viva" (Cahet, 1974: 5).

2) A densidade da macrofauna: atualmente, há um grande interesse em conhecer a densidade do macrobentos das regióes Subantárticas, considerando seu alto teor de biomassa, mas estes estudos são ainda bem limitados. Conforme Dinet et alii 1973 alguns resultados são de delicada comparação, graças aos métodos de coleta e a triagem diferentes.

Dada a importância do conhecimento na-análise da densidade e percentagem da abundância dos principais grupos e de Annelida Polychaeta, em particular, verifica-se que há muito o que precisar, para definir a interação indivíduo-substrato-população e a produtividade.

\section{AGRADECIMENTOS}

Este trabalho foi parte da dissertação do mestrado apresentada à coordenação do Curso de Pós-Graduação em Zoologia da U.F.R.J., para obtenção de grau de Mestre, tendo sido subsidiado pela Universidade Santa Ursula-RJ.

Agradeço à Dra. Jeanete Maron Ramos, orientadora.

\section{REFERENCIAS}

Arnaud, P.M., 1974 - Contribution à la bionomie bentique des régions Antarctiqués et Subantarctiques. Téthys, 6 (3): 465-656.

Bellan, G., 1975. - Polychètes (Serpulidae exclues) des Terres Australes et Antarctiques Françaises. Téthys, 6 (4): 779-798.

Cahet, G., 1974. - Evolution de la matière organique a l'interface: eau-sédiment de milieux margino - littoraux méditerranéens (Golfe du Lion). Thés. Univ. Paris: 1-201.

Chardy, P., D. Desbruyères \& A. Laurec, 1976. Bionomie bentique du plateau continental de l'Archipel des Kerguelen. Macrofaune. (4). Analyse multivariable des taxocénoses Annélidiennes du Golfe du Morbihan. C.N.F.R.A. 39: 97-105.

David, P., J.F. Murail \& Panouse, 1977. - Résultats scientifiques de la Campagne MD 04/ Benthos: hidrologie du plateau continental des Iles Kerguelen. C.N.F.R.A., 42: $41-64$.

De Bovée, F. 1976. - La Nematofaune des vases autopoluées des Ileș Kerguelen (Terres Australes et Antarctiques Françaises) Cah. Biol. Mar., 26 (5): 711-720.

De Bovée, F. \& J. Soyer, 1977. - Le meiobenthos des Iles Kerguelen. Données Quantitatives. I - Le Golfe du Morbihan. II - Le Plateau Continental. III - Conclusions. C.N.F.R.A., 42: 237-265.

Desbruyères, D., 1976a. - Cycle biologique de quelques Annélides Polychètes en milieu SubAntaretique. Paris., Thése Univer. Paris VI, 63 pp.

Desbruyères, D., 1976b. - Bionomie benthique du plateau continental de l'Archipel des Kerguelen. Macrofaune. 3. Contribution à l'étude des Polyonidae (Abbpekudes Oikychètes) des Terres Australes Françaises. C.N.F.R.A. 39: 83-96. 
Desbruyères, D., 1977. - Bionomie bentique du plateau continental des Iles Kerguelen. Macrofaune 6. Evolution des populations de trois espèces d'Annélides Polychètes en Milieu SubAntarctique. C.N.F.R.A., 42: 135-172.

Desbruyères, D., \& A. Guille, 1973. - La faune bentique de 1'Archipel de Kerguelen. Premières donnés quantitatives. C.R. hebd. Séanc. Acad. Sci., Paris, 176:633-636.

Desbruyères, D. \& A. Guille, 1976. - Bionomie bentique du plateau continental des Iles Kerguelen. 8. Variations spatiales et temporelles dans le peuplement des vases à spicules. In: Keega, B.F., P.O.Ceidigh \& P.J.S.Boaden, ed. Biology of Benthic Organisms. Oxford Pergamon: $185-196$.

Dinet, A., L.Laubier, J.Soyer e P.Vitiello, 1973. - Résultats biologiques de la campagne Polymède. II - Le méiobenthos abysal Rapp. Comm. int. Mer. Médit. 21 (9) : 701-704.

Fauvel, P., 1953. - Annélides Polychètes des Iles Kerguelen recueillies par M. Patrice Paulian en 1951. Bull. Inst. Océanogr. Monaco, 1026:1-19.

(iuille, A., 1977 - La campagne MD 04/Benthos du "Marion-Dufresne" (14 février 23 mars 1975): Bionomie du plateau continental des Iles Kerguelen. Station prospectées et résul tats préliminaires. C.N.F.R.A., 42: 3-40.

(iuille, A. \& J. Soyer, 1976. - Prospections bionomiques du plateau Continental des Iles Kerguelen. Golfe du Morbihan et Golfe des Baleiniers. C.N.F.R.A. 39: 49-57.

Aureau, J.C., 1973. - Les possibilités d'exploitation des ressources marines dans les lles Australes Françaises. Bull. Mus. natn. Hist. nat., Paris, Sér. 3, (154), Ecologie générale 10:185191.

Reis, M.A. de C., 1980. - Aglaophamus ornatus Hartman, 1967, (Polychaeta - Nephytidae) da plataforma continental das Ilhas Kerguelen - Região Subantártica. Tese U.F.R.J., 164 pp.

Rullier, F., 1966. - Quelques Annélides Polychètes des Iles Kerguelen. C.N.F.R.A., 32: 1-28. 